\title{
Uncertain Unified Chaotic Systems Control with Input Nonlinearity via Sliding Mode Control
}

\author{
Zhi-ping Shen, ${ }^{1}$ Jian-dong Xiong, ${ }^{1}$ and Yi-lin $\mathrm{Wu}^{2}$ \\ ${ }^{1}$ Henan Engineering Laboratory for Big Data Statistical Analysis and Optimal Control, School of Mathematics and \\ Information Sciences, Henan Normal University, Xinxiang, Henan 453007, China \\ ${ }^{2}$ Department of Computer Science, Guangdong University of Education, Guangzhou, Guangdong 510310, China
}

Correspondence should be addressed to Zhi-ping Shen; zpshen@htu.cn

Received 1 July 2016; Revised 27 August 2016; Accepted 4 September 2016

Academic Editor: Jesus M. Munoz-Pacheco

Copyright (C) 2016 Zhi-ping Shen et al. This is an open access article distributed under the Creative Commons Attribution License, which permits unrestricted use, distribution, and reproduction in any medium, provided the original work is properly cited.

\begin{abstract}
This paper studies the stabilization problem for a class of unified chaotic systems subject to uncertainties and input nonlinearity. Based on the sliding mode control theory, we present a new method for the sliding mode controller design and the control law algorithm for such systems. In order to achieve the goal of stabilization unified chaotic systems, the presented controller can make the movement starting from any point in the state space reach the sliding mode in limited time and asymptotically reach the origin along the switching surface. Compared with the existing literature, the controller designed in this paper has many advantages, such as small chattering, good stability, and less conservative. The analysis of the motion equation and the simulation results all demonstrate that the method is effective.
\end{abstract}

\section{Introduction}

"Chaos" in contemporary English language refers to the meaning of "Unzucht and Unordnung"; its meaning is close to "determinacy for random phenomena" in nonlinear systemic theory. Thus, chaos is borrowed to call these anomalies. In the eyes of appearance, chaos movement looks like random process, but, in fact, there is a distinction between chaotic motion and stochastic process. Chaos has the following features [1]: initial value sensitivity, boundedness, randomness, ergodicity, universality, fractal dimension and positive maximum Lyapunov index, and so forth. It should be pointed out that the identification of chaos is still a topic that is not completely solved till now.

The existence of the chaotic motion can be observed in many cases because chaotic signal has the properties of the inherent continuous broadband power spectra and noise like and so forth. It provides a highly classified secret communication method; chaos control and synchronization and its application in secret communication have attracted many researchers' attention [1-13]. However, due to the fact that the chaotic system is extremely sensitive to initial value and long time unpredictability, chaos control has become the key link of chaotic application. Since 1987, Alekseevv and Loskutov [14] published papers about the controlling chaos, and, in 1989, Hubler et al. [15, 16] proposed OGY method; international and domestic academics have proposed many different methods of chaos control, and the main research results of the chaos control basically have the following categories: feedback control method [17, 18], adaptive control method [19, 20], neural network control method [21], sliding mode control method $[6,22,23]$, and so on.

In 2002, Lü et al. proposed a new chaotic system: the system connects the Lorenz attractor and Chen attractor; Lü system is a special case; hence, it is called the unified chaotic system [24]. Controlling the unified chaotic system had attracted many researchers' attention from the beginning for its special properties; for example, its form is very simple and it only has one parameter, its dynamics behavior can be analyzed using energy barriers principle, it connects the Lorenz attractor and Chen attractor, and it realizes continuous evolution of one system and another system in the whole parameters spectrum. Literature [25] studied the feedback control and synchronization problem of unified chaotic system; literature [26] studied the projective synchronization and control problems of unified chaotic system. In view of 
the system equation containing the unknown parameters, literature [27] studied the stabilization of equilibrium points using the sliding mode variable structure control; literature [28] designed a constraint controller to stabilize the system states to unstable equilibrium points of the unified chaotic system by using the Minimum Principle of Pontryagin, and the paper also presented a combination of Bang-Bang control and logic switching to overcome the limitation of Bang-Bang control; literature [29] proposed a passive equivalent control scheme to realize the stability control of different equilibrium for the unified chaotic system using the passive control theory. Literature [30] proposed a sliding mode controller to synchronize two different chaotic systems with unknown bounded uncertainties. Reference [31] proposed a discontinuous Lyapunov functional approach to achieving asymptotic robust synchronization of uncertain chaotic systems using sampled-data control with stochastically varying sampling intervals. However, there is not an overall and only effective control method in presented methods so far.

This paper studies the stabilization problem of a class of unified chaotic systems with parameter uncertainty and nonlinear input based on the sliding mode variable structure control. A sufficient condition under which the sliding mode system is quadratically stable, a new control method, and the time during which the system states can reach the sliding manifold are given. The designed control law algorithms in this paper and in literature [22] are all able to ensure that the motion of the system can reach the switching surface and thus can make the closed-loop system asymptotically stable, but the control law in paper [22] cannot provide the arrival speed, cannot guarantee rapidity, and had a big conservative, but the control law in this paper can improve the rapidity of the system's trajectories to reach the sliding mode and effectively weaken the chattering of the sliding mode. Simulation results verify that the method is feasible, and the improvement of the dynamic property is prominent.

\section{Problem Formulation}

A class of unified chaotic systems is shown in

$$
\begin{aligned}
& \dot{x}_{1}=(25 \alpha+10)\left(x_{2}-x_{1}\right), \\
& \dot{x}_{2}=(28-35 \alpha) x_{1}+(29 \alpha-1) x_{2}-x_{1} x_{3}, \\
& \dot{x}_{3}=x_{1} x_{2}-\frac{8+\alpha}{3} x_{3},
\end{aligned}
$$

where $\alpha \in[0,1]$, system (1) is always chaotic when $\alpha \in$ $[0,1]$; when $\alpha$ increases from 0 to 1 , system (1) gradually transits from Lorenz system to Chen system. According to the definition in literature [24], system (1) belongs to generalized Lorenz chaotic system when $\alpha \in[0,0.8)$, satisfying $a_{12} a_{21}>$ 0 ; system (1) belongs to generalized Chen chaotic system when $\alpha \in(0.8,1]$, satisfying $a_{12} a_{21}<0$; and when $\alpha=0.8$, system (1) satisfying $a_{12} a_{21}=0$ plays an important role in connecting generalized Lorenz chaotic system to generalized Chen chaotic system. The systems offer the study of chaos control and synchronization of a new mathematical model and make chaos synchronization based on the unified models

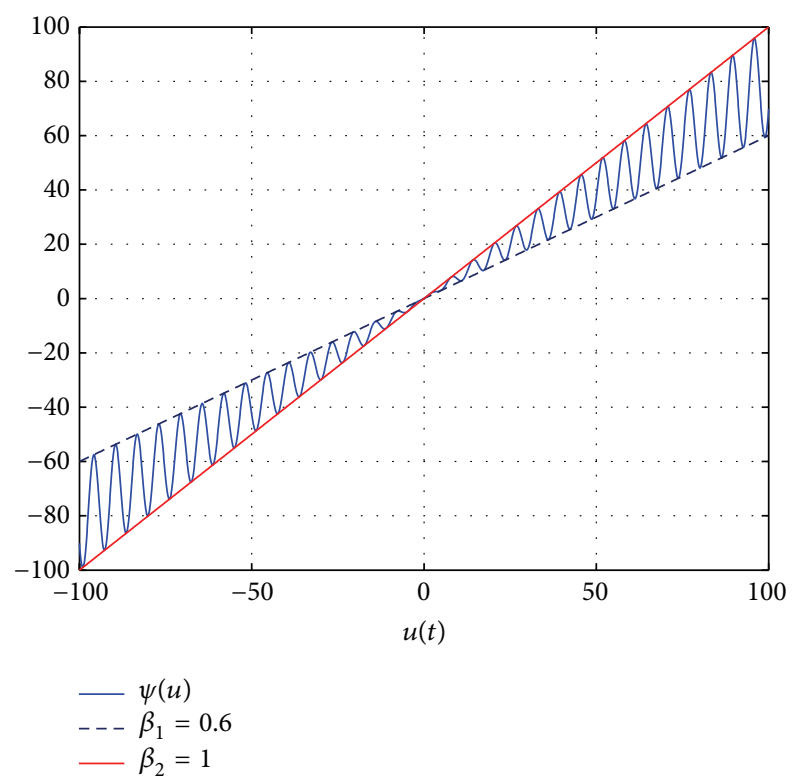

FIGURE 1: Nonlinear function $\psi(u(t))=[0.8+0.2 \sin (u(t))] u(t)$.

that have already gained practical application in secure communications; however, there are still many problems that should be further studied, for example, combining the synchronization methods of the unified chaotic system with the advanced control methods, improving the synchronization performance of a unified chaotic system, and studying nonlinear circuits communication based on unified chaotic system.

The controlled system is as follows:

$$
\begin{aligned}
\dot{x}_{1}= & (25 \alpha+10)\left(x_{2}-x_{1}\right), \\
\dot{x}_{2}= & (28-35 \alpha) x_{1}+(29 \alpha-1) x_{2}-x_{1} x_{3} \\
& +\Delta f\left(x_{1}, x_{2}, x_{3}\right)+\psi(u), \\
\dot{x}_{3}= & x_{1} x_{2}-\frac{8+\alpha}{3} x_{3},
\end{aligned}
$$

where $\Delta f$ is the system uncertainty, satisfying $|\Delta f| \leq \gamma\|x\|, \gamma$ a positive real number, and nonlinear control $\psi(u)$ satisfies

$$
\beta_{1} u^{2} \leq u \psi(u) \leq \beta_{2} u^{2}, \quad 0<\beta_{1}<\beta_{2}
$$

for example,

$$
\psi(u(t))=[0.8+0.2 \sin (u)] u(t)
$$

The figure of the above nonlinear control is shown in Figure 1.

The aim of this paper is to design a controller which can make the closed-loop system stable; therefore, the stabilization problem of system (1) can be transformed into the asymptotic stability problem of system (2); that is,

$$
\lim _{t \rightarrow \infty} x(t)=0
$$




\section{Sliding Mode Design}

Considering the uncertainties in system (2), a sliding mode control method is adopted in this paper to achieve $\lim _{t \rightarrow \infty} x(t)=0$ through its strong robustness on the sliding surface. In order to prove the main results, some lemmas are given.

Lemma 1. If $A$ is a stable and diagonal matrix; then there exists $\lambda$, satisfying

$$
\left\|e^{A t}\right\| \leq e^{-\lambda t} .
$$

Proof. Diagonal matrix A can be rewritten as follows:

$$
A=\operatorname{diag}\left\{\lambda_{1}, \lambda_{2}, \ldots, \lambda_{n}\right\},
$$

where $\lambda_{i}, i=1,2, \ldots, n$ are the diagonal elements and also are eigenvalues of diagonal matrix $A$; let

$$
\lambda_{\max }=\max \left\{\lambda_{1}, \lambda_{2}, \ldots, \lambda_{n}\right\} ;
$$

then, according to the matric exponential function properties, we can get

$$
e^{A t}=\operatorname{diag}\left\{e^{\lambda_{1} t}, e^{\lambda_{2} t}, \ldots, e^{\lambda_{n} t}\right\} ;
$$

thereby,

$$
\left\|e^{A t}\right\|=\left\|\operatorname{diag}\left\{e^{\lambda_{1} t}, e^{\lambda_{2} t}, \ldots, e^{\lambda_{n} t}\right\}\right\| \leq e^{\lambda_{\max } t} .
$$

Let

$$
\lambda=-\lambda_{\max }>0
$$

then,

$$
\left\|e^{A t}\right\| \leq e^{-\lambda t}
$$

Lemma 2. If $A$ is a stable and diagonal matrix and $f(x, t)$ satisfies

$$
\|f(x, t)\| \leq \beta e^{-\zeta t}, \quad \beta>0, \zeta>0,
$$

then the following system is asymptotically stable:

$$
\dot{x}=A x+f(x, t) .
$$

Proof. Solving (14), we can obtain

$$
x(t)=e^{A t} x(0)+\int_{0}^{t} e^{A(t-\tau)} f(x(\tau), \tau) d \tau ;
$$

hence,

$$
\begin{aligned}
\|x(t)\| \leq & \left\|e^{A t}\right\| \cdot\|x(0)\| \\
& +\int_{0}^{t}\left\|e^{A(t-\tau)}\right\| \cdot\|f(x(\tau), \tau)\| d \tau .
\end{aligned}
$$

Let $-\lambda$ be the maximum real part of the eigenvalues of matrix $A$, and from Lemma 1 , we can get

$$
\begin{aligned}
\|x(t)\| & \leq\|x(0)\| e^{-\lambda t}+\left.\frac{\beta}{\lambda-\zeta} e^{-\lambda t} e^{(\lambda-\zeta) \tau}\right|_{0} ^{t} \\
& =e^{-\lambda t}\|x(0)\|+\frac{\beta}{\lambda-\zeta}\left(e^{-\zeta t}-e^{-\lambda t}\right) \longrightarrow 0 .
\end{aligned}
$$

Based on the above lemmas, we shall propose the sliding mode design.

Theorem 3. If we choose the following switching function:

$$
s(t)=-(1-\delta) x_{1}(t)+x_{2}(t), \quad \delta>0,
$$

then, the sliding mode equation is asymptotically stable.

Proof. Choose a switching function as

$$
s(t)=c_{1} x_{1}(t)+c_{3} x_{3}(t)+x_{2}(t),
$$

where $c_{1}, c_{3}$ are parameters to be determined.

On switching surface $s(t)=0$, from (19), we can get

$$
x_{2}(t)=-c_{1} x_{1}(t)-c_{3} x_{3}(t),
$$

substituting (20) to the first and the third equalities of state equation (2); the sliding mode equation is obtained:

$$
\begin{aligned}
& \dot{x}_{1}=-(25 \alpha+10)\left(c_{1}+1\right) x_{1}-(25 \alpha+10) c_{3} x_{3}, \\
& \dot{x}_{3}=-\left(c_{1} x_{1}+c_{3} x_{3}\right) x_{1}-\frac{8+\alpha}{3} x_{3} .
\end{aligned}
$$

The above equation can be rewritten as a vector form:

$$
\left(\begin{array}{l}
\dot{x}_{1} \\
\dot{x}_{3}
\end{array}\right)=\left(\begin{array}{cc}
-\Upsilon\left(c_{1}+1\right) & -\Upsilon c_{3} \\
0 & -\frac{8+\alpha}{3}
\end{array}\right)\left(\begin{array}{l}
x_{1} \\
x_{3}
\end{array}\right)-\left(\begin{array}{l}
0 \\
\bigwedge
\end{array}\right),
$$

where $\Upsilon=(25 \alpha+10), \Lambda=x_{1}\left(c_{1} x_{1}+c_{3} x_{3}\right)$.

Choose $c_{1}=-1+\delta, \delta>0$, and $c_{3}=0$; then (22) becomes

$$
\left(\begin{array}{l}
\dot{x}_{1} \\
\dot{x}_{3}
\end{array}\right)=\left(\begin{array}{cc}
-\Upsilon \delta & 0 \\
0 & -\frac{8+\alpha}{3}
\end{array}\right)\left(\begin{array}{l}
x_{1} \\
x_{3}
\end{array}\right)-\left(\begin{array}{c}
0 \\
\left(c_{1} x_{1}\right) x_{1}
\end{array}\right) .
$$

Solving the first equality of (23), we obtain

$$
x_{1}(t)=x_{1}(0) e^{-(25 \alpha+10) \delta t} \longrightarrow 0 ;
$$

hence, $x_{1}(t)$ is exponentially stable, and because

$$
\left\|\left(c_{1} x_{1}(t)\right) x_{1}(t)\right\| \leq\left\|c_{1} x_{1}(t)\right\| \cdot\left\|x_{1}(t)\right\|,
$$

then $c_{1} x_{1}^{2}(t)$ converges exponentially to zero; thus, from Lemma 2, we obtain

$$
\lim _{t \rightarrow \infty}\left[x_{1}, x_{3}\right]^{T}=0,
$$

which completes the theorem. 


\section{Variable Structure Control Law Design}

Theorem 3 discusses the stability of the system on the switching surface; we now resort to present the sliding controller that can make the movement starting from any point in state space reach the switching surface in limited time.

Theorem 4. If we choose the controller as

$$
u=\frac{1}{2}\left(u^{+}+u^{-}\right)+\frac{1}{2}\left(u^{+}-u^{-}\right) \operatorname{sgn}(s(t))
$$

where

$$
\begin{aligned}
u^{+}= & -\frac{1}{2 \beta_{1}}(|k s|+\epsilon+\Gamma(s, x)+\gamma\|x\|) \\
& -\frac{1}{2 \beta_{1}}|\Gamma(s, x)+\gamma\|x\|+| k s|+\epsilon|, \\
u^{-}= & \frac{1}{2 \beta_{1}}(|k s|+\epsilon-\Gamma(s, x)+\gamma\|x\|) \\
& +\frac{1}{2 \beta_{1}}|-\Gamma(s, x)+\gamma\|x\|+| k s|+\epsilon|, \\
\Gamma(s, x)= & -(1-\delta)(25 \alpha+10)\left(x_{2}-x_{1}\right)-x_{1} x_{3} \\
& +(29 \alpha-1) x_{2}+(28-35 \alpha) x_{1},
\end{aligned}
$$

and parameters $k>0, \epsilon>0$, and $s(t)$ are the switching surface as in (18), then the movement starting from any point in the state space can reach the switching surface in limited time, and the time to reach the switching surface is

$$
T \leq \frac{1}{k} \ln \left(\frac{k}{\epsilon}|s(0)|+1\right) .
$$

Proof. We shall first prove that the movement starting from any point in the state space can reach the switching surface in limited time.

It is easy to verify that (28) and (29) can be rewritten as

$$
\begin{aligned}
& u^{+}=\min \left\{\frac{1}{\beta_{1}}(-|k s|-\epsilon-\Gamma(s, x)-\gamma\|x\|), 0\right\}, \\
& u^{-}=\max \left\{\frac{1}{\beta_{1}}(|k s|+\epsilon-\Gamma(s, x)+\gamma\|x\|), 0\right\} .
\end{aligned}
$$

Because switching surface $s(t)=0$ divides the state space into two parts $s(t)>0$ and $s(t)<0$, we should consider the following two scenarios:

(1) When $s(t)>0$, from (27), we can get $u=u^{+}$, and, then from (32), we can easily get $u=u^{+} \leq 0$, and, since

$$
\beta_{1} u^{2} \leq u \psi(u) \leq \beta_{2} u^{2}, \quad 0<\beta_{1}<\beta_{2},
$$

both sides of the above equation are divided by $u<0$, and we obtain

$$
\beta_{2} u \leq \psi(u) \leq \beta_{1} u
$$

Therefore, by (2), we can get

$$
\begin{aligned}
\dot{s}= & -(1-\delta) \dot{x}_{1}+\dot{x}_{2} \\
= & -(1-\delta)(25 \alpha+10)\left(x_{2}-x_{1}\right)+\Phi x_{1}-x_{1} x_{3} \\
& +(29 \alpha-1) x_{2}+\psi(u)+\Delta f(x, t) \\
\leq & -(1-\delta)(25 \alpha+10)\left(x_{2}-x_{1}\right)+\Phi x_{1}+\beta_{1} u \\
& +(29 \alpha-1) x_{2}-x_{1} x_{3}+|\Delta f(x, t)| \\
\leq & \Gamma(s, x)+\beta_{1} u^{+}+\gamma\|x\| \\
\leq & \Gamma(s, x)+\gamma\|x\|-|k s|-\epsilon-\Gamma(s, x)-\gamma\|x\| \\
= & -|k s|-\epsilon, \quad s>0,
\end{aligned}
$$

where $\Phi=(28-35 \alpha)$.

(2) When $s(t)<0$, from (27), we can get $u=u^{-}$, and, then, from (33), we can easily get $u=u^{-} \geq 0$, so, from (3), we have $\psi(u) \geq \beta_{1} u$, and, then

$$
\begin{aligned}
\dot{s}= & -(1-\delta)(25 \alpha+10)\left(x_{2}-x_{1}\right)+\psi(u)-x_{1} x_{3} \\
& +(28-35 \alpha) x_{1}+(29 \alpha-1) x_{2}+\Delta f(x, t) \\
\geq & -(1-\delta)(25 \alpha+10)\left(x_{2}-x_{1}\right)-x_{1} x_{3}+\beta_{1} u \\
& +\left(28-35 \alpha-x_{3}^{0}\right) x_{1}+(29 \alpha-1) x_{2}-\gamma\|x\| \\
= & \Gamma(s, x)-\gamma\|x\|+\beta_{1} u^{-} \\
\geq & \Gamma(s, x)-\gamma\|x\|+|k s|+\epsilon-\Gamma(s, x)+\gamma\|x\| \\
\geq & |k s|+\epsilon, \quad s<0,
\end{aligned}
$$

from (36) and (37); we can obtain that the movement starting from any point in the state space can reach the switching surface in limited time.

In the sequel, solve the time to reach the switching surface.

For the time to reach the switching surface, through solving (36) and (37), we obtain

$$
\begin{aligned}
& \dot{s} \leq-|k s|-\epsilon, \quad s>0, \\
& \dot{s} \geq|k s|+\epsilon, \quad s<0 .
\end{aligned}
$$

When $s(t)>0$, the first equality in (38) is

$$
\dot{s} \leq-k s-\epsilon .
$$

Letting $w(t)$ be the solution of the following linear differential equation,

$$
\begin{aligned}
& \dot{w}(t)=-k w(t)-\epsilon, \\
& w(0)=s(0) .
\end{aligned}
$$

We conclude by the comparison lemma [32] that

$$
s(t) \leq w(t)=s(0) e^{-k t}-\epsilon \int_{0}^{t} e^{-k(t-\tau)} d \tau .
$$


Similarly, when $s(t)<0$, we have

$$
s(t) \leq s(0) e^{-k t}+\epsilon \int_{0}^{t} e^{-k(t-\tau)} d \tau .
$$

So, we get

$$
\begin{aligned}
& s(t) \leq s(0) e^{-k t}-\epsilon \int_{0}^{t} e^{-k(t-\tau)} d \tau, \\
& s(0)>0, s(t) \geq 0 ; \\
& s(t) \geq s(0) e^{-k t}+\epsilon \int_{0}^{t} e^{-k(t-\tau)} d \tau, \\
& s(0)<0, s(t) \leq 0 .
\end{aligned}
$$

Let the time to reach the switching surface be $T$, since, on switching surface surface $s(t)=0$ and from the above equation, one has

$$
\begin{aligned}
& 0 \leq s(0) e^{-k T}-\frac{\epsilon}{k}\left(1-e^{-k T}\right), \quad s(0)>0, s(t)=0 ; \\
& 0 \geq s(0) e^{-k T}+\frac{\epsilon}{k}\left(1-e^{-k T}\right), \quad s(0)<0, s(t)=0 .
\end{aligned}
$$

Consequently, we have

$$
T \leq \frac{1}{k} \ln \left(\frac{k}{\epsilon}|s(0)|+1\right) .
$$

This completes the proof.

Remark 5. The designed control law algorithms in Theorem 4 and in literature [22] are all able to ensure that the motion of the system can reach the switching surface and hence can make the closed-loop system asymptotically stable, whereas the control law in Theorem 4 has the following advantages:

(1) The designed control law algorithm in literature [22] can only guarantee $s(t) \dot{s}(t)<0$ and cannot provide arrival speed and cannot guarantee rapidity, while the designed control law algorithm in Theorem 4 can guarantee

$$
\begin{aligned}
& \dot{s} \leq-|k s|-\epsilon, \quad s>0, \\
& \dot{s} \geq|k s|+\epsilon, \quad s<0,
\end{aligned}
$$

and the time to reach switching surface $T$ is

$$
T \leq \frac{1}{k} \ln \left(\frac{k}{\epsilon}|s(0)|+1\right) ;
$$

hence, the system has good rapidity.

(2) The designed control law algorithm in literature [22] uses $|\Gamma(s, x)|$, while, in Theorem $4, \Gamma(s, x)$ is employed, which results in small chattering, good stability, and less conservative.

\section{An Improved Control Law}

The designed control law algorithm in Theorem 4 only limits the minimum speed in which the movement starting from any point in state space reaches the switching surface:

$$
|\dot{s}| \geq|k s|+\epsilon
$$

but does not limit the maximum speed, which may result in too fast to reach the switching surface, as a result, the sliding mode control may cause severe chattering, for example,

when $s>0$, and

$$
\begin{aligned}
\Gamma(s, x)+\gamma\|x\| & \ll-|k s|-\epsilon, \\
-|k s|-\epsilon-\Gamma(s, x)-\gamma\|x\|>0, &
\end{aligned}
$$

« denoting "much less than," we have $u=u^{+}=0$, and from (27) and (32), one has

$$
\dot{s} \leq \Gamma(s, x)+\gamma\|x\| \ll-|k s|-\epsilon, \quad s>0 .
$$

Similarly, when $s<0$, and

$$
\begin{aligned}
\Gamma(s, x)-\gamma\|x\| & \gg|k s|+\epsilon, \\
0 & >|k s|+\epsilon-\Gamma(s, x)+\gamma\|x\|,
\end{aligned}
$$

we have $u=u^{-}=0$, and from (27) and (33), one has

$$
\dot{s} \geq \Gamma(s, x)-\gamma\|x\| \gg|k s|+\epsilon, \quad s<0 .
$$

Equations (50) and (52) indicate that the controller designed by (27)-(30) may lead to

$$
\begin{aligned}
& \dot{s} \ll-|k s|-\epsilon, \quad s>0, \\
& \dot{s} \gg|k s|+\epsilon, \quad s<0 ;
\end{aligned}
$$

that is to say, the movement starting from any point in the state space reaches the switching surface too fast, which may cause that the sliding mode produces severe chattering and the system dynamic quality is poor. In order to avoid these disadvantages, we shall make the following improvement for the control law in Theorem 4; choose

$$
\begin{aligned}
u_{*}^{+}= & -\frac{1}{2}\left(\frac{1}{\beta_{1}}+\frac{1}{\beta_{2}}\right)(|k s|+\epsilon+\Gamma(s, x)+\gamma\|x\|) \\
& -\frac{1}{2}\left(\frac{1}{\beta_{1}}-\frac{1}{\beta_{2}}\right)|\Gamma(s, x)+\gamma\|x\|+| k s|+\epsilon|, \\
u_{*}^{-}= & -\frac{1}{2}\left(\frac{1}{\beta_{1}}+\frac{1}{\beta_{2}}\right)(-|k s|-\epsilon+\Gamma(s, x)-\gamma\|x\|) \\
& -\frac{1}{2}\left(\frac{1}{\beta_{2}}-\frac{1}{\beta_{1}}\right)|\Gamma(s, x)-\gamma\|x\|-| k s|-\epsilon|,
\end{aligned}
$$

which is to say that we shall present the following improved controller theorem which can avoid the above disadvantages.

Theorem 6. If we choose the controller as

$$
u=\frac{1}{2}\left(u_{*}^{+}+u_{*}^{-}\right)+\frac{1}{2}\left(u_{*}^{+}-u_{*}^{-}\right) \operatorname{sgn}(s(t)),
$$

then the movement starting from any point in the state space can reach the switching surface in limited time, and the time to reach the switching surface is

$$
T \leq \frac{1}{k} \ln \left(\frac{k}{\epsilon}|s(0)|+1\right) .
$$

Meanwhile, the movement starting from any point in the state space reaches the switching surface not too fast. 
Proof. Consider the following four scenarios.

(1) When $s>0$ and $|k s|+\epsilon+\Gamma(s, x)+\gamma\|x\|>0$, we have

$$
u_{*}^{+}=\frac{1}{\beta_{1}}(-|k s|-\epsilon-\Gamma(s, x)-\gamma\|x\|)<0 ;
$$

hence, from the switching surface equation, we obtain

$$
\begin{aligned}
\dot{s} & \leq \Gamma(s, x)+\beta_{1} u_{*}^{+}+\gamma\|x\| \\
& \leq \Gamma(s, x)+\gamma\|x\|-|k s|-\epsilon-\Gamma(s, x)-\gamma\|x\| \\
& \leq-k|s|-\epsilon .
\end{aligned}
$$

(2) When $s>0$ and $|k s|+\epsilon+\Gamma(s, x)+\gamma\|x\|<0$, we have

$$
u=u_{*}^{+}=-\frac{1}{\beta_{2}}(|k s|+\epsilon+\Gamma(s, x)+\gamma\|x\|)>0 .
$$

From (3), we get $\psi(u) \leq \beta_{2} u$, substituting it into the switching surface equation; we obtain

$$
\begin{aligned}
\dot{s} & \leq \Gamma(s, x)+\psi(u)+\gamma\|x\| \leq \Gamma(s, x)+\beta_{2} u_{*}^{+}+\gamma\|x\| \\
& \leq \Gamma(s, x)+\gamma\|x\|-|k s|-\epsilon-\Gamma(s, x)-\gamma\|x\| \\
& \leq-|k s|-\epsilon .
\end{aligned}
$$

(3) When $s<0$ and $-|k s|-\epsilon+\Gamma(s, x)-\gamma\|x\|>0$, we get

$$
u=u_{*}^{-}=\frac{1}{\beta_{2}}(|k s|+\epsilon-\Gamma(s, x)+\gamma\|x\|)<0 .
$$

From (3), we get $\psi(u) \geq \beta_{2} u$, substituting it into the switching surface equation, and we obtain

$$
\begin{aligned}
\dot{s} & \geq \Gamma(s, x)+\psi(u)-\gamma\|x\| \geq \Gamma(s, x)+\beta_{2} u_{*}^{-}-\gamma\|x\| \\
& \geq \Gamma(s, x)-\gamma\|x\|+|k s|+\epsilon-\Gamma(s, x)+\gamma\|x\| \\
& \geq|k s|+\epsilon .
\end{aligned}
$$

(4) When $s<0$ and $-|k s|-\epsilon+\Gamma(s, x)-\gamma\|x\|<0$, we get

$$
u_{*}^{-}=\frac{1}{\beta_{1}}(|k s|+\epsilon-\Gamma(s, x)+\gamma\|x\|)>0 .
$$

From the above equation, we get $\psi(u) \geq \beta_{1} u$, substituting it into the switching surface equation, and we obtain

$$
\begin{aligned}
\dot{s} & \geq \Gamma(s, x)+\psi(u)-\gamma\|x\| \geq \Gamma(s, x)+\beta_{2} u_{*}^{-}-\gamma\|x\| \\
& \geq \Gamma(s, x)-\gamma\|x\|+|k s|+\epsilon-\Gamma(s, x)+\gamma\|x\| \\
& \geq|k s|+\epsilon ;
\end{aligned}
$$

hence, we can obtain Theorem 6 from (58)-(64).

Comparison between the Control Law in Theorems 4 and 6. From the proofs of Theorems 4 and 6, we can see that when

$$
\begin{array}{ll}
s>0, & -|k s|-\epsilon-\Gamma(s, x)-\gamma\|x\|<0, \\
s<0, & -|k s|-\epsilon+\Gamma(s, x)-\gamma\|x\|>0,
\end{array}
$$

the speed of reaching the switching surface is obtained by the control laws in Theorem 4, and its expression is

$$
\begin{aligned}
& \dot{s} \leq \Gamma(s, x)+\beta_{1} u^{+}+\gamma\|x\|=-|k s|-\epsilon, \quad s>0, \\
& \dot{s} \geq \Gamma(s, x)+\beta_{2} u^{-}-\gamma\|x\|=|k s|+\epsilon, \quad s<0,
\end{aligned}
$$

and the speed of reaching the switching surface is obtained by the control laws in Theorem 6, and its expression is

$$
\begin{aligned}
& \dot{s} \leq \Gamma(s, x)+\beta_{1} u_{*}^{+}+\gamma\|x\|=-|k s|-\epsilon, \quad s>0, \\
& \dot{s} \geq \Gamma(s, x)+\beta_{2} u_{*}^{-}-\gamma\|x\|=|k s|+\epsilon, \quad s<0,
\end{aligned}
$$

and we can see that the speeds obtained by the two control laws are identical from the above two equations, but, when

$$
\begin{array}{ll}
s>0, & \Gamma(s, x)+\gamma\|x\| \ll-|k s|-\epsilon, \\
s<0, & \Gamma(s, x)-\gamma\|x\| \gg|k s|+\epsilon,
\end{array}
$$

by the control law in Theorem 4 , we get

$$
u=u^{-}=u^{+}=0 .
$$

Substituting the above controller into the switching surface equation, we get

$$
\begin{aligned}
& \dot{s} \leq \Gamma(s, x)+\beta_{1} u+\gamma\|x\| \ll-|k s|-\epsilon, \quad s>0, \\
& \dot{s} \leq \Gamma(s, x)+\beta_{2} u-\gamma\|x\| \gg|k s|+\epsilon, \quad s<0,
\end{aligned}
$$

while, by the control law in Theorem 6 , we get

$$
u=u_{*}^{+}=u_{*}^{-}=-\frac{|k s|+\epsilon+\Gamma(s, x)+\gamma\|x\|}{\beta_{2}}>0 .
$$

Substituting the above controller into the switching surface equation, we get

$$
\begin{aligned}
& \dot{s} \leq \Gamma(s, x)+\beta_{2} u_{*}^{+}+\gamma\|x\|=-|k s|-\epsilon, \quad s>0, \\
& \dot{s} \geq \Gamma(s, x)+\beta_{2} u_{*}^{-}-\gamma\|x\|=|k s|+\epsilon, \quad s<0,
\end{aligned}
$$

and we can see that the improved control law can change the case that the movement starting from any point in the state space reaches the switching surface too fast from (70) and (72). We can improve the rapidity of the system's trajectories to reach the sliding mode, effectively weaken the chattering of sliding mode, and improve the system dynamic quality by choosing appropriate parameters $k>0$ and $\epsilon>0$.

\section{Simulation Example}

In this section, we shall give an example to demonstrate the effectiveness of the control law in Theorem 6 and meanwhile compare it with the control law in literature [22].

When $\alpha=1$, the unified chaotic system (1) denotes Chen's chaotic system, that is

$$
\begin{aligned}
& \dot{x}_{1}=35\left(x_{2}-x_{1}\right), \\
& \dot{x}_{2}=-7 x_{1}+28 x_{2}-x_{1} x_{3}, \\
& \dot{x}_{3}=x_{1} x_{2}-3 x_{3},
\end{aligned}
$$




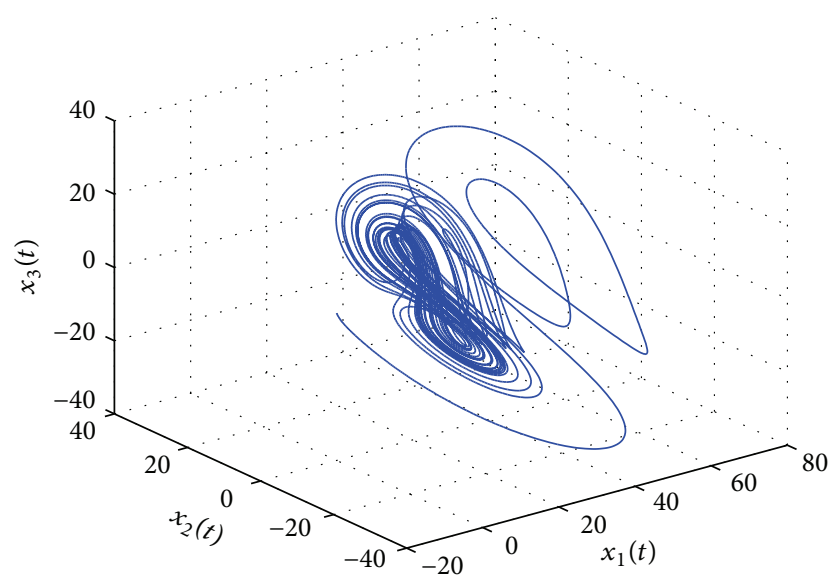

FIgURE 2: Phase portraits of Chen chaotic system.

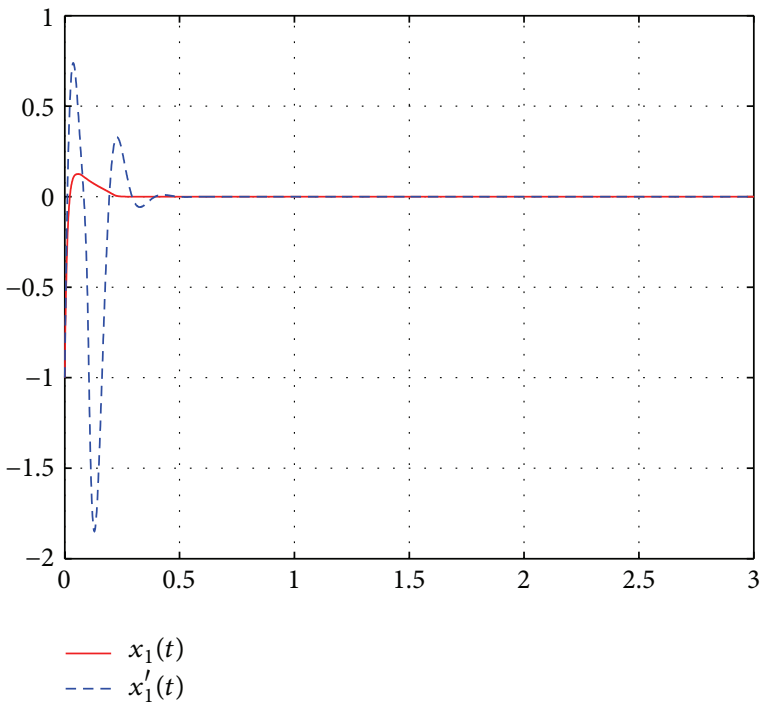

FIGURE 3: State variable $x_{1}(t)-t$.

by using Simulink, and Chen's chaotic attractor is shown in Figure 2, where we choose the initial values as $\left(x_{1}(0)\right.$, $\left.x_{2}(0), x_{3}(0)\right)=(1.00,-1.00,-1.01)$.

The controlled system with disturbances is

$$
\begin{aligned}
& \dot{x}_{1}=35\left(x_{2}-x_{1}\right), \\
& \dot{x}_{2}=-7 x_{1}+28 x_{2}-x_{1} x_{3}+\Delta f(x, t)+\psi(u), \\
& \dot{x}_{3}=x_{1} x_{2}-3 x_{3},
\end{aligned}
$$

where external disturbance $\Delta f=0.5 \cos (3 \pi t)\|x\|, x=$ $\left(x_{1}, x_{2}, x_{3}\right)^{T}$.

Obviously, parameter $\gamma=0.5$. We choose nonlinear input as $\psi(u(t))=[0.8+0.2 \sin (u(t))] u(t)$; from (3), we can get parameters $\beta_{1}=0.6$ and $\beta_{2}=1.0$. Simulation results of nonlinear function $\psi(u(t))$ are shown in Figure 1.

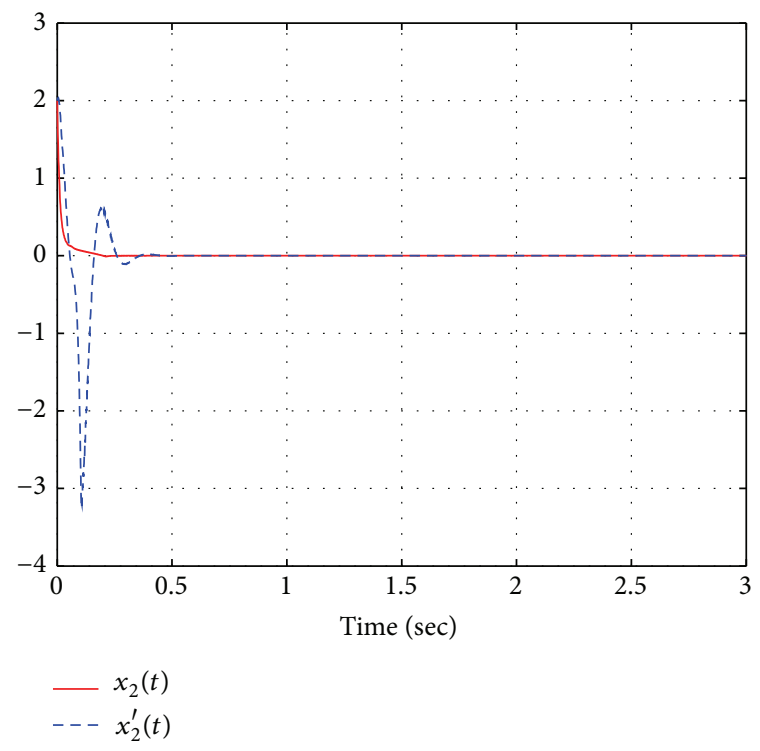

Figure 4: State variable $x_{2}(t)-t$.

6.1. Simulation Example by Using This Paper Control Law. According to Theorem 3 in this paper, we can choose the switching surface function as

$$
s(t)=-(1-\delta) x_{1}+x_{2},
$$

where parameter $\delta=2$, and, according to (55) in Theorem 6 and (30) and (54), we can choose the control law as

$$
u(t)=\frac{1}{2}\left(u_{*}^{+}+u_{*}^{-}\right)+\frac{1}{2}\left(u_{*}^{+}-u_{*}^{-}\right) \operatorname{sgn}(s(t)),
$$

where $k=1, \epsilon=0.5, c_{1}=\delta-1=1$ are chosen.

6.2. Simulation Example by Using the Control Law in Paper [22]. According to (5) and (6) in literature [22], we can choose the corresponding switching surface function as

$$
\begin{aligned}
s(t) & =x_{2}(t)+\rho(t), \\
\dot{\rho}(t) & =35 x_{1}+x_{1} x_{3}+0.5 x_{2},
\end{aligned}
$$

and, according to (11) in Theorem 1 in literature [22], we can choose the control law as

$$
\begin{aligned}
u(t) & =-2 \eta \operatorname{sgn}(s(t)), \\
\eta & =\left|28 x_{1}+27 x_{2}\right|+0.5\|x\| .
\end{aligned}
$$

Simulink results are shown in Figures 3-7, where the red solid lines in all curves denote Simulink results obtained by the control law in this paper, the blue dashed lines in all curves denote Simulink results obtained by the control law in paper [22], which is to say, $x_{1}(t), x_{2}(t), x_{3}(t), s(t), u(t)$ denote Simulink results obtained by the control law in this paper and $x_{1}^{\prime}(t), x_{2}^{\prime}(t), x_{3}^{\prime}(t), s^{\prime}(t), u^{\prime}(t)$ denote Simulink results obtained by the control law in paper [22] in all curves.

Figures 3-5 are the state curves with respect to time; we can see that the designed control law algorithm in this paper 


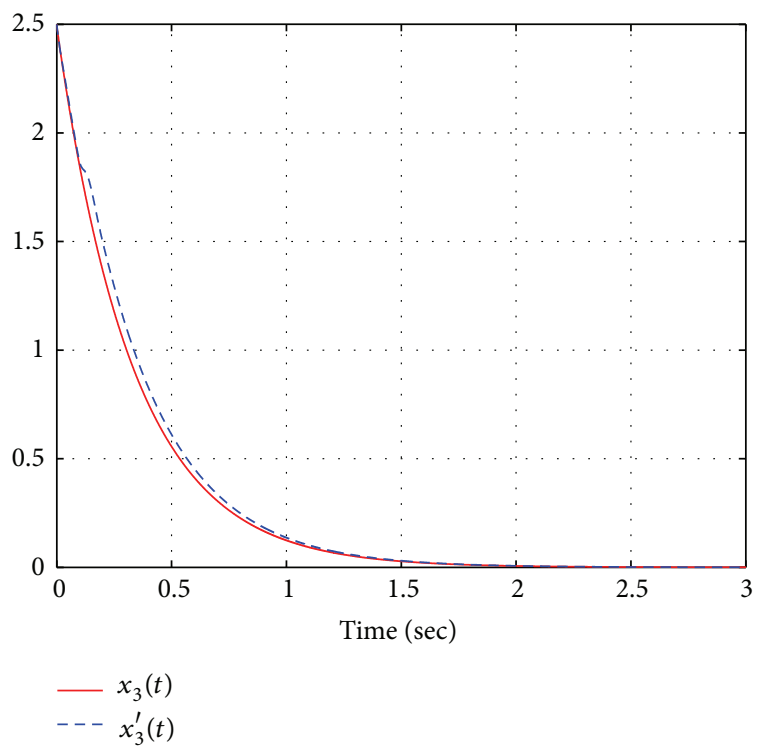

Figure 5: State variable $x_{3}(t)-t$.

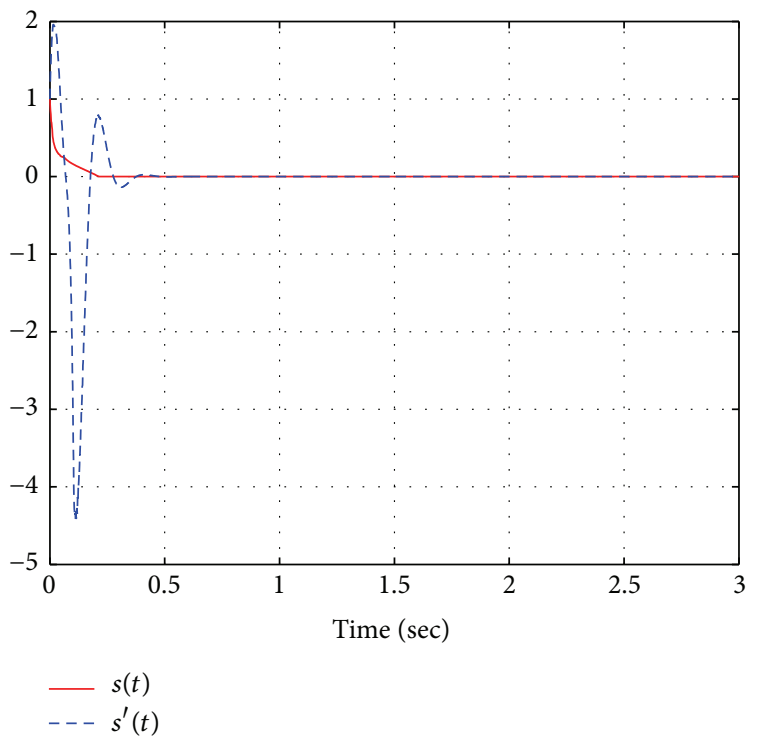

FIGURE 6: Sliding mode surface $s(t)-t$.

and in literature [22] are all able to control the state variable to the neighborhood of the equilibrium point, but the solid lines (the designed control law algorithm in this paper) are superior to the dashed lines (the control law in paper [22]) in rapidity and stationarity. Figure 6 is the switching function curve with respect to time, and it can be seen that the control law in this paper can effectively weaken the chattering of the sliding mode, the chattering obtained by the designed control law algorithm in this paper is much less than the chattering obtained by the designed control law algorithm in paper [22]. Figure 7 is the control function curve with respect to time, as can be seen by comparing; the controller designed in this paper has many advantages, such as small chattering, good stability, and less conservative.

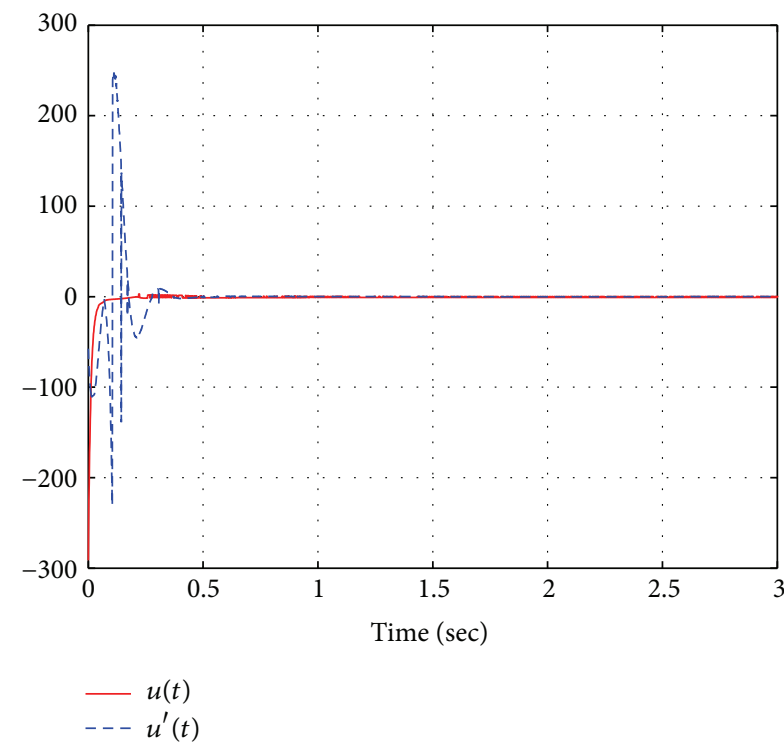

Figure 7: Input variable $u(t)-t$.

\section{Conclusions}

The designed control law algorithm in this paper can realize the stabilization of the chaotic system with external disturbances and has good inhibition to the uncertainty. Compared with the existing literature, the control law algorithm in this paper can improve the quickness of reaching the sliding mode and also effectively weaken the chattering of the sliding mode; meanwhile the algorithm also can reduce the conservatism of the parameter design of the controller, and both the analysis of the motion equation and simulation results confirm the effectiveness of the conclusion.

\section{Competing Interests}

The authors declare that they have no competing interests.

\section{Acknowledgments}

This work was supported by National Natural Science Foundation of China (60774057); Appropriative Researching Fund for Professors and Doctors, Guangdong University of Education (2014ARF25); Science and Technology Planning Project of Guangdong Province, China (2016A010106007); Applied Science and Technology Special Funds Project of Guangdong Province, China (2016B090927010); Foundation for Ph.D. of Henan Normal University, China (5101019170158); Key Scientific Research Project of Universities and Colleges in Henan, China (16A120005).

\section{References}

[1] F. H. Min, Research Some Problems in Control and Synchronization of Chaotic Systems, Nanjing University of Science and Technology, Nanjing, China, 2007. 
[2] S.-Y. Li and Z.-M. Ge, "Fuzzy modeling and synchronization of two totally different chaotic systems via novel fuzzy model," IEEE Transactions on Systems, Man, and Cybernetics, Part B: Cybernetics, vol. 41, no. 4, pp. 1015-1026, 2011.

[3] M. Srivastava, S. P. Ansari, S. K. Agrawal, S. Das, and A. Y. Leung, "Anti-synchronization between identical and nonidentical fractional-order chaotic systems using active control method," Nonlinear Dynamics, vol. 76, no. 2, pp. 905-914, 2014.

[4] L. Zhang and X. Liu, "The synchronization between two discrete-time chaotic systems using active robust model predictive control," Nonlinear Dynamics, vol. 74, no. 4, pp. 905-910, 2013.

[5] X. Yang, J. Cao, and J. Lu, "Synchronization of markovian coupled neural networks with nonidentical node-delays and random coupling strengths," IEEE Transactions on Neural Networks and Learning Systems, vol. 23, no. 1, pp. 60-71, 2012.

[6] M. Yahyazadeh, N. R. Noei, and R. Ghaderi, "Synchronization of chaotic systems with known and unknown parameters using a modified active sliding mode control," ISA Transactions, vol. 50, no. 2, pp. 262-267, 2011.

[7] X. Feng, F. Zhang, and W. Wang, "Global exponential synchronization of delayed fuzzy cellular neural networks with impulsive effects," Chaos, Solitons \& Fractals, vol. 44, no. 1-3, pp. 9-16, 2011.

[8] J. Lu, D. W. C. Ho, J. Cao, and J. Kurths, "Exponential synchronization of linearly coupled neural networks with impulsive disturbances," IEEE Transactions on Neural Networks, vol. 22, no. 2, pp. 329-335, 2011.

[9] W. He, F. Qian, Q.-L. Han, and J. Cao, "Synchronization error estimation and controller design for delayed Lur'e systems with parameter mismatches," IEEE Transactions on Neural Networks and Learning Systems, vol. 23, no. 10, pp. 1551-1563, 2012.

[10] W. Zhou, D. Tong, Y. Gao, C. Ji, and H. Su, "Mode and delay dependent adaptive exponential synchronization in pth moment for stochastic delayed neural networks with Markovian switching," IEEE Transactions on Neural Networks and Learning Systems, vol. 23, no. 4, pp. 662-668, 2012.

[11] Z.-G. Wu, P. Shi, H. Su, and J. Chu, "Exponential synchronization of neural networks with discrete and distributed delays under time-varying sampling," IEEE Transactions on Neural Networks and Learning Systems, vol. 23, no. 9, pp. 1368-1376, 2012.

[12] H.-J. Zhu and C.-B. Zeng, "Scaling and mixed synchronization for different chaotic systems with totally unknown parameters," Control Theory \& Applications, vol. 32, no. 3, pp. 341-346, 2015.

[13] Z.-B. Li and J.-S. Tang, "Chaotic synchronization with parameter perturbation and its secure communication scheme," Control Theory \& Applications, vol. 31, no. 5, pp. 592-600, 2014.

[14] V. V. Alekseevv and A. Y. Loskutov, "Control of a system with a strange attractor through periodic parametric action," Soviet Physics Doklady, vol. 32, no. 4, pp. 1346-1348, 1987.

[15] A. W. Hubler, "Adaptive control of chaotic systems," Helvetica Physica Acta, vol. 62, no. 2, pp. 343-346, 1989.

[16] E. Ott, C. Grebogi, and J. A. Yorke, "Controlling chaos," Physical Review Letters, vol. 64, no. 11, pp. 1196-1199, 1990.

[17] J. A. Gallegos, "Nonlinear regulation of a Lorenz system by feedback linearization techniques," Dynamics and Control, vol. 4, no. 3, pp. 277-298, 1994.

[18] X.-S. Luo, B.-H. Wang, F. Jiang, and Y. Gao, "Using random proportional pulse feedback of system variables to control chaos and hyperchaos," Chinese Physics, vol. 10, no. 1, pp. 17-20, 2001.
[19] G.-G. Liu and Y. Zhao, "Adaptive control on a class of uncertain chaotic systems," Chinese Physics Letters, vol. 22, no. 5, pp. 10691071, 2005.

[20] R. Luo and Y. Zeng, "The adaptive control of unknown chaotic systems with external disturbance via a single input," Nonlinear Dynamics, vol. 80, no. 1-2, pp. 989-998, 2015.

[21] C.-T. Lin and C.-P. Jou, "Controlling chaos by GA-based reinforcement learning neural network," IEEE Transactions on Neural Networks, vol. 10, no. 4, pp. 846-859, 1999.

[22] T.-Y. Chiang, M.-L. Hung, J.-J. Yan, Y.-S. Yang, and J.-F. Chang, "Sliding mode control for uncertain unified chaotic systems with input nonlinearity," Chaos, Solitons \& Fractals, vol. 34, no. 2, pp. 437-442, 2007.

[23] L. G. Zhang and Y. Yan, "Robust synchronization of two different uncertain fractional-order chaotic systems via adaptive sliding mode control," Nonlinear Dynamics, vol. 76, no. 3, pp. 1761-1767, 2014.

[24] J. Lü, G. Chen, D. Cheng, and S. Celikovsky, "Bridge the gap between the Lorenz system and the Chen system," International Journal of Bifurcation and Chaos, vol. 12, no. 12, pp. 2917-2926, 2002.

[25] C.-H. Tao, J.-A. Lu, and J.-H. Lü, "The feedback synchronization of a unified chaotic system," Acta Physica Sinica, vol. 51, no. 7, pp. 1500-1501, 2002.

[26] J. Liu, S. H. Chen, and J. A. Lu, "Projective synchronization in a unified chaotic system and its control," Acta Physica Sinica, vol. 52, no. 7, pp. 1595-1599, 2003.

[27] X.-P. Guan and Y.-H. He, "Stabilizing unstable equilibrium point of unified chaotic systems with unknown parameter using sliding mode control," Chinese Physics Letters, vol. 21, no. 2, pp. 227-229, 2004.

[28] Y. Z. Song, G. Z. Zhao, and D. L. Qi, "Some comments on constrained control of chaos," Physics Letters A, vol. 359, no. 6, pp. 624-626, 2006.

[29] D.-L. Qi, X.-R. Li, and G.-Z. Zhao, "Passive control of hybrid chaotic dynamical systems," Journal of Zhejiang University, vol. 38, no. 1, pp. 86-97, 2004.

[30] J. T. Li, W. L. Li, and Q. P. Li, "Sliding mode control for uncertain chaotic systems with input nonlinearity," Communications in Nonlinear Science and Numerical Simulation, vol. 17, no. 1, pp. 341-348, 2012.

[31] T. H. Lee, J. H. Park, S. M. Lee, and O. M. Kwon, "Robust synchronisation of chaotic systems with randomly occurring uncertainties via stochastic sampled-data control," International Journal of Control, vol. 86, no. 1, pp. 107-119, 2013.

[32] K. K. Hassan, Nonlinear Systems, Prentice Hall, 3rd edition, 2001. 


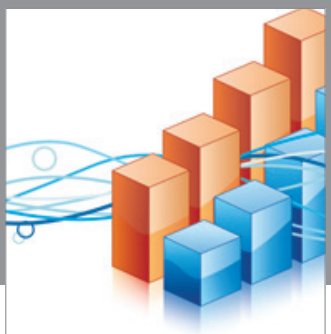

Advances in

Operations Research

vatem alat4

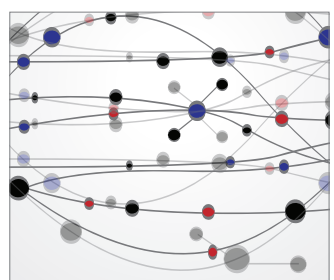

\section{The Scientific} World Journal
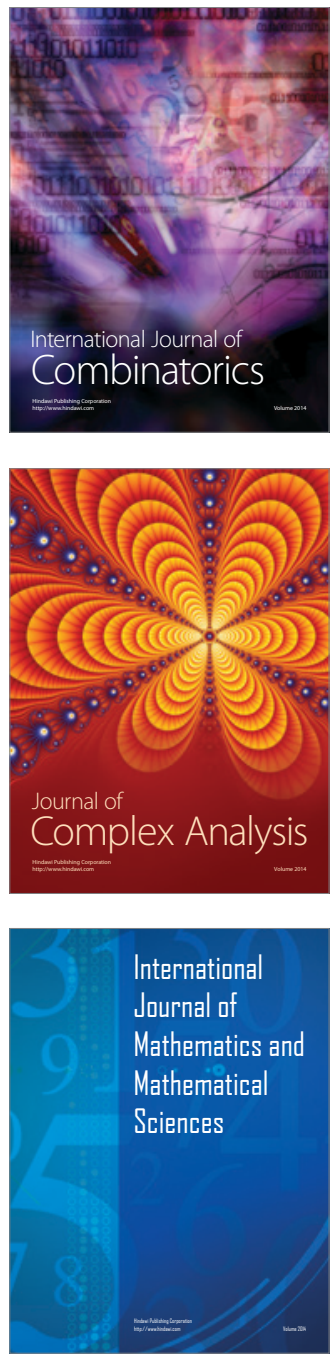
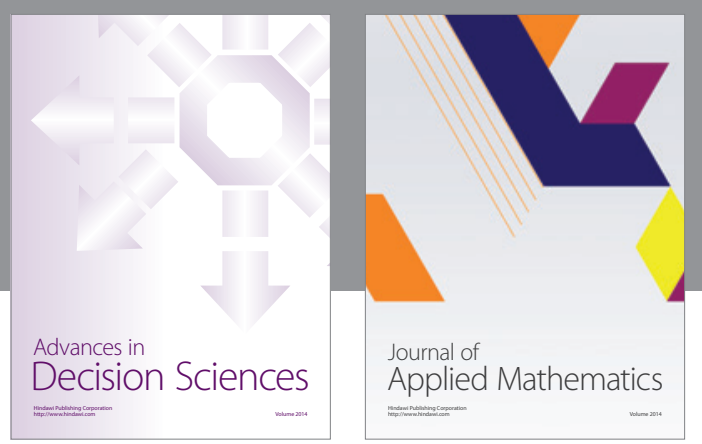

Algebra

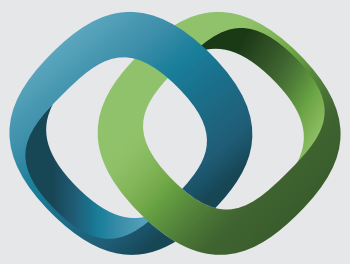

\section{Hindawi}

Submit your manuscripts at

http://www.hindawi.com
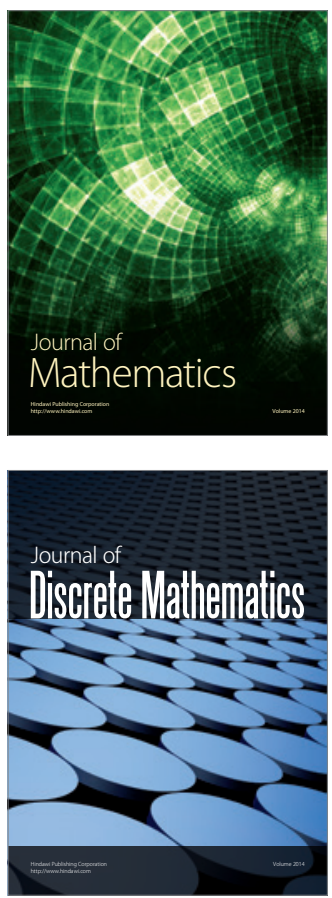

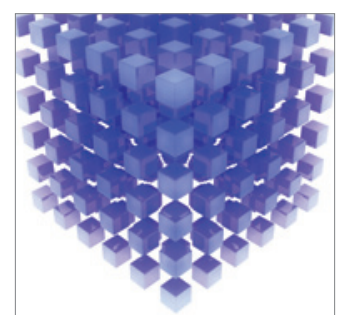

Mathematical Problems in Engineering
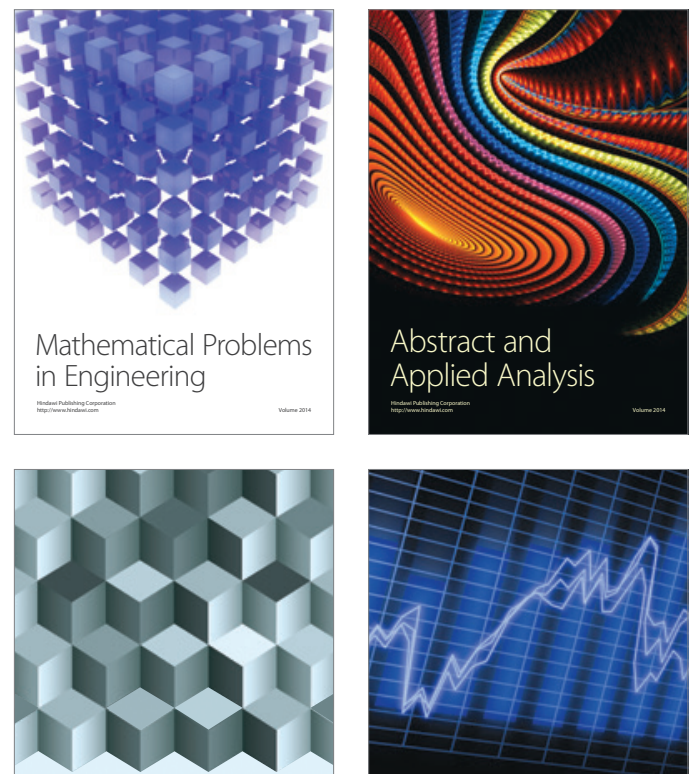

Journal of

Function Spaces

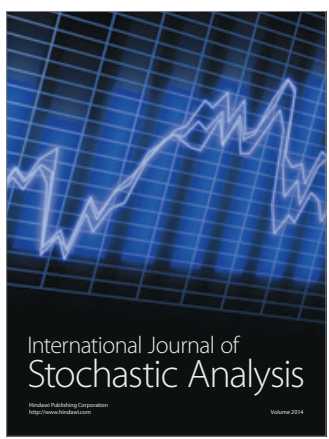

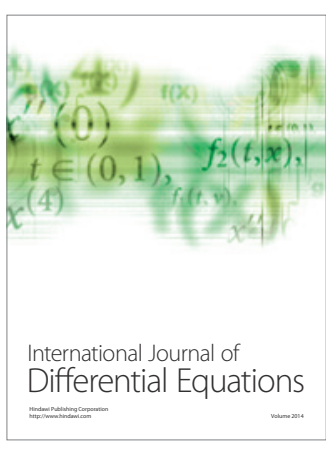
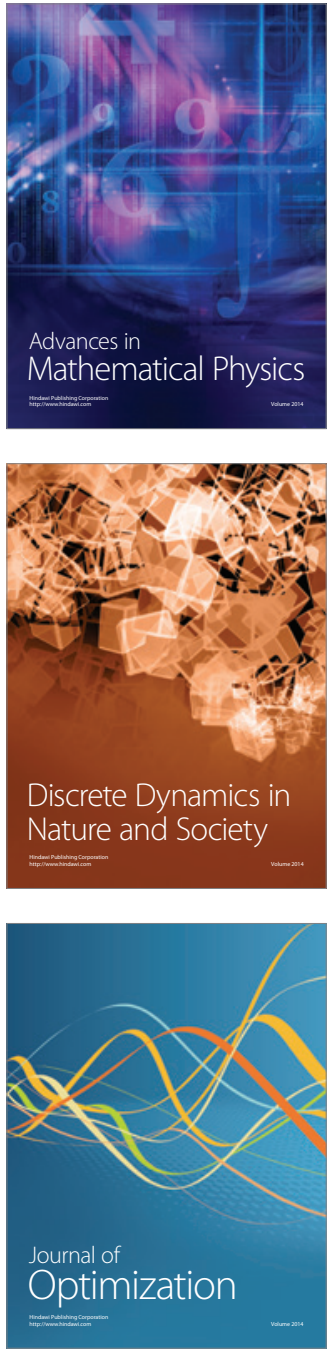